\title{
Skopje à l'époque des Jeunes-Turcs
}

Skopje in the Young Turks era

Petar Todorov

\section{OpenEdition}

\section{Journals}

Édition électronique

URL : https://journals.openedition.org/ceb/1087

DOI : $10.4000 /$ ceb. 1087

ISSN : 2261-4184

Éditeur

INALCO

\section{Édition imprimée}

Date de publication : 9 janvier 2012

ISSN : 0290-7402

\section{Référence électronique}

Petar Todorov, « Skopje à l'époque des Jeunes-Turcs », Cahiers balkaniques [En ligne], 40 | 2012, mis en ligne le 25 mai 2012, consulté le 06 juillet 2021. URL : http://journals.openedition.org/ceb/1087 ; DOI : https://doi.org/10.4000/ceb.1087

Ce document a été généré automatiquement le 6 juillet 2021

\section{(c) (†) 8}

Cahiers balkaniques est mis à disposition selon les termes de la Licence Creative Commons Attribution - Pas d'Utilisation Commerciale 4.0 International. 


\title{
Skopje à l'époque des Jeunes-Turcs
}

\author{
Skopje in the Young Turks era
}

\section{Petar Todorov}

1 En juillet 1908, à Ferizovik, les chefs albanais ont manifesté contre les ingérences étrangères dans leur région. Cette réunion, autour de Shemsi Pacha, utilisée, voire organisée par les autorités ottomanes, et ensuite instrumentalisée par le comité jeuneturc de Skopje, a été un facteur important dans le retour de la Constitution de $1876^{1}$. On voit ainsi l'importance des élites albanaises comme facteur politique dans le vilayet du Kosovo, et dans son centre administratif, Skopje. On peut dire que, dans ce contexte, la politique et l'existence du mouvement des Jeunes-Turcs ont été souvent liées à la position des Albanais locaux.

2 La proclamation de la Constitution a été accueillie dans l'euphorie par toutes les communautés, à Skopje, comme dans les autres centres urbains. Mais comment les citoyens de Skopje l'ont-ils interprétée et quelles étaient leurs espérances? L'ont-ils tous réellement accepté?

3 La région de Skopje, comme tout le Kosovo en général, était fidèle au Sultan Abdul Hamid II, ce qui poussait les musulmans, majoritairement des Albanais, à critiquer les interventions des Puissances en faveur des chrétiens et renforçait l'identité religieuse des Albanais locaux. Aussi, lors de la réunion de Ferizovik, les Jeunes-Turcs, pour convaincre les élites albanaises d'accepter la Constitution, doivent-ils promettre qu'elle dérive de la charî'a. De cette approche naîtront des problèmes par la suite. Certains musulmans ont vu la Constitution comme un moyen d'arrêter les réformes conduites dans l'Empire, Galib bey (le commandant de la gendarmerie réorganisée) par exemple, pendant les premiers jours, a changé son nouvel uniforme pour l'ancien, comme un signe de refus des réformes ${ }^{2}$. Les chefs albanais aussi, immédiatement après le retour de la Constitution, ont exigé l'arrêt des réformes. Un document concernant le kaza de Preshevo, proche de Skopje, nous informe que les musulmans de cette région ont vu dans la Constitution une opportunité de devenir des maîtres, tandis que les chrétiens seraient leurs serviteurs ${ }^{3}$. 
4 Au même moment, les chrétiens voyaient la Constitution comme la fin du régime d'oppression du Sultan Abdul Hamid. En même temps, ils espéraient qu'elle allait arrêter les luttes entre les propagandistes des pays voisins, ainsi qu'entre les chrétiens et les musulmans. Le but ultime, pensaient-ils, était l'égalité avec les musulmans, c'est à dire, une plus grande participation des chrétiens dans l'administration, et c'est précisément le non-accomplissement de ces espérances qui sera, plus tard, le fond de leur mécontentement contre les Jeunes-Turcs. Un facteur décisif dans leurs positions fut leur appartenance aux différents groupes religieux et organisations politiques, dont certaines suivaient la politique des pays voisins, la Serbie et la Bulgarie.

5 Dans ces conditions nouvelles, les communautés ont présenté leurs intérêts devant les autorités ottomanes à travers les clubs et les partis politiques. Ces clubs ont été abolis avec la loi sur les associations de 1909, mais leurs activités se sont poursuivies, à travers l'Église et les écoles. Les activités des "cheta " liées aux différentes communautés chrétiennes ont également diminué, mais n'ont pas cessé malgré leur dissolution après la proclamation de la Constitution. Les acteurs politiques les plus importants chez les musulmans de Skopje étaient le Comité des Jeunes-Turcs « Union et Progrès » (par la suite, le Comité), et le club albanais. Outre la division entre exarchistes et patriarchistes serbes, les chrétiens étaient également organisés en plusieurs organisations politiques. Les patriarchistes serbes étaient membres du club serbe ou de la Ligue Démocratique Serbe (parti politique serbe), fondée à Skopje. De leur côté, les exarchistes étaient divisés en deux organisations rivales, le Club Constitutionnel Bulgare et une section de la Partie Fédérative Populaire (PFP). Un club grec a aussi existé dans la ville, mais, en raison du petit nombre de Grecs, il n'avait presque aucune activité ${ }^{4}$. On note également un club hébraïque qui était fidèle au Comité jeune-turc. La proclamation de la Constitution a aussi donné la possibilité de créer des organisations syndicales et socialistes, mais hormis l'organisation de grèves et la première célébration du $1^{\mathrm{er}}$ Mai (où il n'y eut que 50-60 participants), leur activité resta limitée, en raison du sousdéveloppement de l'industrie et du manque d'ouvriers dans la région.

\section{Les Albanais et le mouvement des Jeunes-Turcs}

\section{L'attachement au sultan}

6 Parmi les dirigeants du mouvement jeune-turc à Skopje, en majorité des officiers de l'armée ottomane et des notables turcs, on trouve également un certain nombre d'Albanais. Parmi eux, on note Nexhip Draga, membre renommé du Comité à Skopje, et Sali Bey, un haut fonctionnaire jeune-turc. Le premier a eu le mérite de convaincre les Albanais de Ferizovik d'accepter la Constitution, le second a changé de parti et pendant le mouvement contre constitutionnel à Istanbul, s'est mis à la tête de l'opposition contre les Jeunes-Turcs à Skopje. Pour mieux comprendre la position des Albanais ${ }^{5}$, il faut savoir que le développement du nationalisme albanais dans cette région était encore faible et que l'identité religieuse était dominante. La grande majorité était attachée à la politique et la personne du Sultan Abdul Hamid II. Certains chefs albanais avaient largement profité de concessions spéciales de la part du Sultan, on comprend donc pourquoi le changement de sultan après l'échec du mouvement contre constitutionnel n'a pas été accueilli avec plaisir à Skopje. Les Jeunes-Turcs ont dû faire campagne pour dénoncer les crimes d'Abdul Hamid et de son régime ${ }^{6}$. À l'égard des 
Jeunes-Turcs, on rencontre chez les chefs albanais deux lignes principales : un groupe complètement opposé qui demandait le retour à la charî'a a et un deuxième groupe qui, tout en restant opposé, soutenait la Constitution en tant que facteur d'ordre.

Dans les jours et les semaines précédant la proclamation de la Constitution la plupart des citoyens de Skopje ont été en faveur des Jeunes-Turcs, et certains les ont rejoints, les uns sous la menace, les autres par opportunisme, y compris parmi des personnes jusque-là adversaires du mouvement. On connaît les noms de certains d'entre eux : Mahmud Shefket Pasha (vali du Vilayet de Kosovo) ${ }^{7}$, Vasfi bey (militaire), Izet bey (militaire), Hadi Pasha (commandant de la division de Skopje). Juste après la proclamation, ils sont devenus les plus grands partisans de la Constitution, provoquant une division entre les nouveaux membres ${ }^{8}$ et les anciens dont certains, mécontents, sont passés dans l'opposition contre le Comité.

À Istanbul, l'opposition se concentrait dans le Parti Libéral et les groupes religieux. Les libéraux ont insisté sur la décentralisation de l'Empire et l'égalité entre les confessions religieuses, ce que soutenaient les non-musulmans. Les religieux ont demandé le retour à la charî'a. À Skopje l'opposition la plus forte venait des religieux; les autres opposants étaient les anciens membres mécontents du Comité dont les dirigeants principaux étaient les deux chefs albanais déjà mentionnés, Sali bey et Nexhip Draga. Une raison supplémentaire au mécontentement des Albanais, outre les fausses promesses concernant la Sheria, était la politique des Jeunes-Turcs envers eux. Contrairement à Abdul Hamid, ils voulaient en effet incorporer les Albanais au sein de l'ottomanisme, préserver leur loyauté et les mettre sous son contrôle?. Les révoltes dans les parties septentrionales du Vilayet de Kosovo en 1910 et surtout en 1911 ont été une conséquence importante de cette politique.

\section{Le mouvement anticonstitutionnel}

9 Comme l'année précédente, en 1909 aussi le soutien albanais était jugé important par les Jeunes-Turcs, cette fois pour protéger la Constitution. Ils se sont donc efforcés de conserver la faveur des Albanais et de les écarter du mouvement anticonstitutionnel à Istanbul ${ }^{10}$.

On ne voit pas de mouvement anticonstitutionnel fort à Skopje avant l'arrivée des nouvelles de la capitale. À ce moment-là les hodjas et certains notables albanais se sont prononcés contre la constitution dans les mosquées et demandèrent le retour de la charî'a. Même Sali Bey, un défenseur libéral de la Constitution, a justifié cela en disant qu'on avait promis aux Albanais que la Constitution dériverait de la charî'a ${ }^{11}$. Ce personnage a joué, malgré le rôle important des religieux, un rôle crucial dans les événements d'avril 1909 à Skopje, car il défendait la Constitution, mais critiquait fortement la politique des Jeunes-Turcs. La décision finale des Albanais et des musulmans en général, envers la Constitution a été prise dans une réunion à Skopje, ${ }^{12}$ organisée par les Jeunes-Turcs, comme l'année précédente à Ferizovik. Ils ont inclus, de nouveau, la charî'a dans leurs discours, utilisant une lettre du Grand Vizir, qui recommandait aux habitants de Skopje le respect pour la charî'a comme pour la Constitution. Cela a provoqué des réactions positives parmi les Albanais, qui ont voté une fois de plus pour la Constitution ${ }^{13}$.

11 Le résultat de ces événements à Skopje a été de consolider le pouvoir des Jeunes-Turcs. Les partisans de l'ancien régime ont été relevés de leurs fonctions. Cependant, le 
mécontentement envers le Comité n'a pas cessé; au contraire, il s'est graduellement agrandi dans la période suivante. En raison de la destitution du Sultan Abdul Hamid, l'anniversaire de la Constitution est célébré sans grande solennité, et cela jusqu'à la fin de la période ottomane; de plus, une grande partie de la population est restée indifférente. Le Comité n'a pas bénéficié d'un soutien important ni des musulmans, ni des chrétiens. En 1911, Nexhip Draga, un membre de longue date, quitte le Comité et tente d'organiser une opposition contre son ancienne organisation. Il soutient Said Hodja, membre de l'Assemblée ottomane en visite à Skopje, pour convaincre d'autres membres de quitter le Comité ${ }^{14}$. Dans cette lutte entre les Jeunes-Turcs et la nouvelle opposition, les Albanais ont joué de nouveau un rôle important. Ainsi, le journal de l'opposition a planifié de s'appuyer sur eux ${ }^{15}$.

12 En général, les Jeunes-Turcs ont voulu conserver la fidélité des Albanais, en utilisant les méthodes d'Abdul Hamid. C'est pourquoi les unités militaires de Skopje n'ont presque pas participé dans la campagne contre le mouvement anticonstitutionnel à Istanbul. Les Jeunes-Turcs ont également utilisé des Albanais dans la politique contre le VMRO. En février 1909, comme l'année suivante, le Comité a décidé de créer des unités albanaises pour lutter contre les « cheta » de VMRO ${ }^{16}$. Les relations entre le Comité et les Albanais touchent aussi la question de l'utilisation de la langue albanaise dans les écoles d'État: en 1910 la classe d'albanais a été fermée, tandis que les élèves étaient transférés dans l'école d'Elbasan.

\section{Les communautés chrétiennes face à la nouvelle situation politique}

\section{Les relations entre les Jeunes-Turcs et les organisations politiques chrétiennes}

13 Les relations entre les Jeunes-Turcs et les communautés chrétiennes, comme avec les Albanais, étaient liées à la politique de l'ottomanisme. Il s'agit dans ce cas, de la question des écoles et des églises, et la dissolution des "cheta " en Macédoine, y compris à Skopje. Une autre question était le problème agricole, souvent mis en avant par le PFP. Les facteurs extérieurs prirent aussi une place importante, en particulier les relations avec la Bulgarie et la Grèce. Les Jeunes-Turcs, comme leurs prédécesseurs, ont choisi de soutenir le plus faible contre le plus fort.

Pendant les premiers mois du pouvoir constitutionnel à Skopje, on n'a pas noté de détérioration des relations entre le Comité et les communautés chrétiennes. Une petite exception a été un malentendu insignifiant entre le Comité et les Serbes : les JeunesTurcs ayant interprété les réserves serbes comme une attitude anticonstitutionnelle, les Serbes prirent des mesures pour prouver le contraire ${ }^{17}$. L'intérêt principal de Serbes était la reconnaissance d'un millet serbe dans l'Empire. Les autorités ottomanes n'ont jamais accepté cette demande, bien qu'elles aient inclus parfois ce problème dans leurs discours à Skopje. L'organisation serbe n'a jamais cessé de chercher à réaliser son programme national, ce qui était contraire aux idées des Jeunes-Turcs.

Dans les relations entre le Comité et les exarchistes on note différentes positions en raison de l'existence de deux groupes exarchistes, le Club Constitutionnel Bulgare et le PFP. Les relations entre le Comité et le Club Constitutionnel Bulgare étaient bonnes au 
début, ils étaient même parvenus à un compromis pour une liste commune aux élections parlementaires ${ }^{18}$, mais cet accord a été rompu par la proclamation de l'indépendance de la Bulgarie. C'est pourquoi les Jeunes-Turcs ont cherché le soutien du PFP contre le Club Constitutionnel. Ces organisations exarchistes étaient dans une rivalité permanente. Les relations avec le PFP étaient, elles aussi, compliquées et les Jeunes-Turcs ont mené envers lui une politique ambiguë ${ }^{19}$. Le Club et le PFP sont issus des divisions internes du VMORO. Le club était dirigé par Hristo Matov, ancien membre et chef du district révolutionnaire de Skopje du VMORO. Le PFP était dirigé par Petar Pop Arsov, lui aussi ancien membre du VMORO. Dans l'historiographie macédonienne, les premiers sont connus comme des "neovrhovisti», tandis que les autres, supporteurs de Jane Sandanski, sont connus comme des «sandanisti». Dans la configuration politique nouvelle, les partisans de Matov se sont organisés assez vite dans le Club Constitutionnel Bulgare, sous l'influence directe de Sofia. Les partisans de Sandanski se sont organisés dans le PFP. Les sandanistes de Skopje, pour la plupart, ont lié leur politique à celle des Jeunes-Turcs pour la défense de la Constitution et contre l'influence étrangère. Un autre soutien important des Jeunes-Turcs a été Jordan Shurkov. Maintes fois, dans ses discours publics, il a soutenu les Jeunes-Turcs et critiqué les organisations nationales aidées par les pays voisins ${ }^{20}$. Il est même devenu membre, avec d'autres du PFP, du Parti Démocratique Ottoman. Mais ce parti et ses membres chrétiens ont été considérés comme traîtres par la majorité des chrétiens ${ }^{21}$. Hors des projets des Jeunes-Turcs, on trouve aussi Petar Pop Arsov ${ }^{22}$. La tentative des Jeunes-Turcs pour rallier les chrétiens de Skopje à leur politique d'ottomanisme a échoué.

Le conflit entre le PFP et le Club Constitutionnel à Skopje a duré jusqu'à la contrerévolution à Istanbul et la décision du PFP de défendre la Constitution et les JeunesTurcs. À cause de cela, le PFP a perdu son influence à Skopje, et bientôt (en 1910) le parti a été dissous. Le Club Constitutionnel et les patriarchistes serbes sont restés neutres pendant ces événements, et du même coup sont devenus les plus importants facteurs politiques chrétiens dans la ville.

17 Assez vite, les organisations principales des chrétiens ont changé d'attitude envers les Jeunes-Turcs. En fait, au contraire de l'ottomanisme, elles ont accentué leur identité nationale, dans ce cas, serbe et bulgare, menant la lutte dans les écoles et les églises, puisqu'elles ont dû limiter les activités des «chetas » armées. Tout de suite après la proclamation de la Constitution les Jeunes-Turcs avaient insisté pour une dissolution rapide de ces « chetas ». L'ont-elles été vraiment?

Chaque organisation craignait une attaque imminente de l'autre et aucune n'a donc dissous sa "cheta ", contrairement aux discours pacifistes, le désarmement n'a pas été effectif $^{23}$. Quelques mois à peine après la proclamation de la Constitution, on note la présence des « chetas » dans les environs de Skopje. Les consuls serbes et français nous informent de la présence d'une cheta près du village de Kozle (décembre 1908) et d'une autre qui parcourt la région (février 1909). Seul changement: leur fonction n'est plus d'attaquer les autorités ottomanes, mais de maintenir la discipline dans l'organisation et le statu quo dans le partage des villages. 


\section{Les réactions de la population chrétienne et la tentative de fraternisation entre les communautés}

Un aspect important dans les textes concernant les Jeunes-Turcs en Macédoine, souvent négligé par l'historiographie macédonienne, est la réaction du peuple. Une grande partie des chrétiens a accepté de participer au travail des nouveaux comités et des tribunaux. Mais en réalité ces gens étaient traités comme des membres passifs, invités seulement pour entériner les décisions déjà prises par le Comité. Ce fut la première déception des chrétiens face aux Jeunes-Turcs ${ }^{24}$. Ceux-ci, en fait, craignaient les réactions des musulmans qui attendaient le renforcement de leur pouvoir et n'ont pas cherché à rétablir l'égalité. Cependant, les paysans chrétiens qui avaient beaucoup souffert des chetas et voulaient vivre en paix, ont beaucoup apprécié la décision jeuneturque de les supprimer, on note même dans des rapports des cas où ils ont chassé les chetas de leur village ${ }^{25}$.

La cohabitation pacifique entre les communautés et la fin des combats a tenu une place importante dans les discours du nouveau pouvoir. L'intolérance précédente était telle que parfois les membres d'une communauté refusaient de fréquenter les cafés de la communauté rivale. La pacification et la fraternisation ont été visibles au début de la période constitutionnelle à Skopje, comme dans les autres villes. Les chefs des chetas, anciens ennemis, ont déposé les armes et célébré la Constitution, les différents groupes ont lutté ensemble de manière exemplaire contre le grand incendie dans le charsija en août $1908^{26}$.

21 Mais ces exemples resteront uniques. En réalité, la pacification tant souhaitée n'a jamais été réalisée. Des attaques de part et d'autre sont déjà notées dans les mois suivant la proclamation. En août 1908 ont lieu les premiers incidents entre les exarchistes et les patriarchistes serbes, en mars 1909 a lieu le premier assassinat politique, un chef exarchiste est tué parce qu'il est passé dans le camp adverse ${ }^{27}$. Il y a eu également des attaques entre chrétiens et musulmans. Les rapports consulaires nous signalent des meurtres d'Albanais commis par des chrétiens ${ }^{28}$. Dans la période suivante, les conflits entre les groupes religieux et politiques à Skopje ne vont pas cesser de croitre.

Les raisons de l'échec de la pacification sont multiples. Premièrement, la position géographique de Skopje et la lutte entre les exarchistes et les patriarchistes serbes pour dominer la région. Deuxièmement, l'incapacité des Jeunes-Turcs à résoudre les problèmes politiques et les programmes nationaux. Comme leurs prédécesseurs, ils ont répondu aux conflits religieux et politiques en armant les musulmans, ce qui n'a pas contribué à la pacification.

\section{Conclusion}

Dans la période suivante, à Skopje, comme dans tout le vilayet du Kosovo, des manifestations contre les Jeunes-Turcs auront lieu. En 1910 et 1911, la ville vit avec les événements du vilayet (les révoltes des Albanais), et les actions terroristes de district révolutionnaire (du VMRO) de Skopje, menées par Hristo Matov et Todor Alexandrov. 


\section{BIBLIOGRAPHIE}

Cette étude est fondée quasi exclusivement sur un travail d'archives.

On peut citer deux ouvrages de référence.

Clayer, Nathalie, (2007), Aux origines du nationalisme albanais : la naissance d'une nation majoritairement musulmane en Europe, Paris : Karthala/CERI, coll. « Recherches internationales ».

Pandevski, Manol, (1987), ПОЛИТИЧКИТЕ ПАРТИИ И ОРГАНИЗАЦИИ ВО МАКЕДОНИЈА (1908-1912) [Les Partis et Organisations politiques en Macédoine (1908-1912)], Skopje.

\section{NOTES}

1. Nathalie Clayer, Aux origines du nationalisme albanais: La naissance d'une nation majoritairement musulmane en Europe, Paris, 2007. p. 607.

2. Archives Nationales de la République de Macédoine (ANRM), Le ministère des Affaires étrangères - section politique et éducative. M-817, Rapport du Consul Balugdjic, 24/07/1908. Cet acte était suivi par les autres officiers dans la ville.

3. ANRM, M-818, Balugdjic, 01/08/1908.

4. Une communauté valaque qui d'après différentes sources comptait environ 250 membres.

5. Nathalie Clayer, op.cit. p. 686.

6. ANRM, M-462, Balugdjic, 01/05/1909.

7. Mahmud Shefket Pasha après la proclamation de la Constitution devient le commandant du III Corps d'Armée.

8. ANRM, M-817, Balugdjic, 31/07/1908, 01/08/1908, 05/08/1908, 13/11/1908; Archive du Ministère des Affaires étrangères (AMAE) Nantes, Consulat Salonique - Serie B, carton 13 , Krajewski, 28/07/1908.

9. Nathalie Clayer, op.cit.p.616.

10. ANRM, M-462, Balugdjic 17/04/1909, 19/04/1909 ; AMAE - Nantes, Krajewski.17/04/1909.

11. ANRM, M-462, Balugdjic, 16/04/1909.

12. La réunion avait été convoquée par le comité des Jeunes-Turcs. Représentants de toutes les communautés dans la ville étaient présents.

13. ANRM, M-462, Balugdjic, 16/04/1909.

14. ANRM, M-878, Jovanovic, 22/09/1911.

15. ANRM, M-878, Jovanovic, 25/09/1911.

16. AMAE - Nantes, Krajewski, 20/02/1909, 09/10/1910.

17. ANRM, Balugdjic, 30/07/1908.

18. Manol Pandevski, ПОЛИТИЧКИТЕ ПАРТИИ И ОРГАНИЗАЦИИ ВО МАКЕДОНИЈА (1908-1912), Les Partis et Organisations politiques en Macédoine (1908-1912), Skopje 1987, p. 117.

19. Ibidem, p. 119.

20. ANRM, M-832, Balugdjic, 03/07/1909, M-840, Balugdjic, 13/07/1909.

21. AMAE - Nantes, Calvière, 10/12/1909.

22. ANRM, M-840, Balugdjic, 13/07/1909.

23. Le Comité des Jeunes-Turcs a organisé encore une action de désarmement, cette fois de toute la population. Le désarmement de la population dans le caza de Skopje s'est fait avec succès ce qui n'est pas cas dans les autres cazas du sandjak de Skopje. Après ce désarmement, les Jeunes- 
Turcs, très vite, ont commencé à armer la population musulmane. D’après les autorités, cette action devait protéger les musulmans des actions des chetas bulgares.

24. ANRM, M-818, Balugdjic, 07/08/1908.

25. AMAE - Nantes, Calvière, 19/11/1910, Vanco Gorgiev, Petar Pop Arsov, Skopje 1997. p. 133.

26. AMAE - Nantes, Krajewski, 11/08/1908, ANRM, M-821, Balugdjic, 10/08/1908.

27. AMAE - Nantes, Krajewski, 18/03/1908.

28. AMAE - Nantes, Krajewski, 14/03/1908, ANRM, M-832, Balugdjic, 07/06/1909.

\section{RÉSUMÉS}

À Skopje, les chrétiens comme ailleurs dans l'Empire accueillent favorablement les Jeunes-Turcs ; en revanche, les musulmans, le plus souvent des Albanais, sont assez hostiles, attachés au sultan, craignant de s'éloigner de la charî'a et peu désireux de donner des pouvoirs aux chrétiens. Ceuxci d'ailleurs ne dépassent pas leurs divisions entre «nations » et obédiences religieuses, ce qui limite donc la « fraternité » annoncée, puisqu'aucun groupe ne dissout ses bandes armées.

In Skopje, as nearly everywhere in the Ottoman Empire Christians populations greeted the Young Turks movement with enthusiasm. On the contrary, the muslims, most often Albanians in this case, are rather hostile, from commitment to the Sultan, and not wishing to move away from the serya or give powers to the Christians. Moreover, the Christians themselves are not able to go over their divisions between «nations » and religious obediences, which restrains the promised fraternity, as no group disbands its armed militia.

ВО СКОПЈЕ, КАКО И ВО ОСТАНАТИОТ ДЕЛ ОД ИМПЕРИЈАТА, ХРИСТИЈАНИТЕ ПОСИТИВНО ГИ ПРИФАГЈААТ МЛАДОТУРЦИТЕ; ОД ДРУГА СТРАНА, МУСЛИМАНИТЕ НАЈЦЕСТО АЛБАНЦИТЕ, СЕ ПОНИПРИЈАТЕЛСКИ РАСПОЛОГЕНИ И ПРИВРЗАНИ КОН СУЛТАНОТ ПЛАЧЕЧИ СЕ ДА СЕ ОДАЛЕЧАТ ЧЕРИЈАТОТ ТИЕ НЕ СЕ РАСПОЛОГЕНИ ДА ДАДАТ ВЛАСТ НА ХРИСТИЈАНИТЕ. ИСТОВРЕМЕНО ТИЕ НЕ ГИ НАДМИНУВААТ НИВНИТЕ ПОДЕЛБИ “ПОМЕГЈУ НАЦИИ И РЕЛИГИОЗНА ПОКОРНОСТ, ШТО ГО ПРЕШУВА ПОГЛАСЕНОТО "БРАТСТВО", СО ОГЛЕД НА ТОА ШТО НИТУ ЕДНА СО ЗАЕДНИЦИТЕ НЕ ГИ РАСПУШТА ЦЕЛОСНО СВОИТЕ ВООРУ'ЕНИ ШЕТИ.

\section{INDEX}

Index géographique : Ferizovik, Kosovo, Macédoine, Skopje

motsclestr Ferizovik, Kosova, Krousovo, Makedonya, Osmanlı İmparatorluğu, Üsküb

motsclesmk АЛБАНИЈА, МЛАДОТУРСКАТА, КОСОВО

Thèmes : Histoire

Index chronologique : Empire ottoman, révolution jeune-turque (1908-1914)

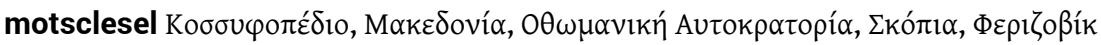

Mots-clés : Caza/Kaza, Çete/chette/tchete/tsete, Exarchat, Jeunes-Turcs, ORIM/VRMO, Sandanski Yané (1872-1915), Vali, Vilayet, albanais

Keywords : Young Turks, Albanian, Shari'a, Macedonia, Skopje, Ferizovik, Kossovo, Ottoman empire, Young Turks revolution (1908-1914), History 